\title{
Hydrogenated Amorphous Silicon Carbide Double Graded-Gap p-i-n Thin-Film Light-Emitting Diodes
}

\author{
Nerng-Fu Shin, Jyh-Young Chen, Tean-Sen Jen, Jyh-Wong Hong, \\ and Chun-Yen Chang, Fellow, IEEE
}

\begin{abstract}
Hydrogenated amorphous silicon carbide (a-SiC:H) p-i-n thin-film light-emitting diodes (TFLED's) with graded $p^{+}-i$ and $\mathrm{i}-\mathrm{n}^{+}$junctions have been proposed and fabricated successfully on an indium-tin-oxide (ITO)-coated glass. An obtained orange TFLED reveals a brightness of $207 \mathrm{~cd} / \mathrm{m}^{2}$ at an injection current density of $600 \mathrm{~mA} / \mathrm{cm}^{2}$, which is the brightest one ever reported for a-SiC:H TFLED's at the same injection current density. This significant increase of brightness could be ascribed to the combined effect of reduced interface states by using the graded-gap junctions, lower contact resistance due to postmetallization annealing, and higher optical gaps of the doped layers.
\end{abstract}

\section{INTRODUCTION}

$\mathrm{E}$ LECTROLUMINESCENCE (EL) studies of amorphous semiconductors have been done by several research groups over the past few years [1]-[8]. As compared with conventional crystal light-emitting diodes (LED's), the potential applications of hydrogenated amorphous silicon carbide (a-SiC:H) thin-film LED's (TFLED's) show several advantages, such as accessibility to large-area flat-panel displays, easy to deposit onto any substrate without the problem of lattice match, and lower cost. The obtainable brightness of a-SiC:H TFLED so far, however, is still insufficient for practical applications. This is partially due to the limitation of the valency-electron controllability of high optical-gap a-SiC:H [6], [7]. In this work, to enhance the EL intensity, a new device structure of double graded-gap (DG) a-SiC:H p-i-n TFLED is proposed, fabricated, and characterized.

\section{DEVICE FABRICATION}

After cleaning, ITO-coated Corning 7059 glass substrates were put into the reaction chamber of a ULVAC CPD-1108D plasma-enhanced chemical vapor deposition (PECVD) system for a 2 -h heat-cleaning process at a substrate temperature of $180^{\circ} \mathrm{C}$ and in a vacuum of $4 \times$ $10^{-6}$ torr. Then a $\mathrm{H}_{2}$-plasma bombarding to the ITO

\footnotetext{
Manuscript received March 8, 1993; revised June 22, 1993. This work was supported by the National Science Council of the Republic of China under Contract NSC 81-0417-E008-548.

N. -F. Shin, J. -Y. Chen, T. -S. Jen, and J. -W. Hong are with the Department of Electrical Engineering, National Central University, Chungli, Taiwan, 32054, Republic of China.

C. -Y. Chang is with the Institute of Electronics, National Chiao-Tung University, Hsinchu, Taiwan, 30050, Republic of China. IEEE Log Number 9211601
}

surface was used to reduce the contact resistance between the ITO electrode and $\mathrm{p}^{+}$-layer [9]. In order to obtain higher optical-gap layers, the $\mathrm{p}^{+}$-type (or $\mathrm{n}^{+}$-type) layer was deposited by feeding $1 \% \mathrm{~B}_{2} \mathrm{H}_{6} 72 \mathrm{sccm}$ (or $1 \% \mathrm{PH}_{3}$ $144 \mathrm{sccm}$ ), pure $\mathrm{C}_{2} \mathrm{H}_{2} 6 \mathrm{sccm}$, and $4 \% \mathrm{SiH}_{4} 200 \mathrm{sccm}$ into the chamber [4], [10]. The i-type layers were deposited by feeding $4 \% \mathrm{SiH}_{4} 200 \mathrm{sccm}$ and pure $\mathrm{C}_{2} \mathrm{H}_{2} 10 \mathrm{sccm}$ for the Type I device and $16 \mathrm{sccm}$ for the Type II device into the chamber. The Type II device was used to study the influence of wider optical-gap i-layer. The substrate temperature, chamber pressure, and RF power density were kept at $180^{\circ} \mathrm{C}, 0.3$ torr, and $16 \mathrm{~mW} / \mathrm{cm}^{2}$ during various film depositions, respectively. The contact resistances between the $\mathrm{p}^{+}$-layer and the ITO with and without $\mathrm{H}_{2}$ plasma treatment are $7.22 \times 10^{5}$ and $2.42 \times 10^{5} \Omega \cdot \mathrm{cm}^{2}$, respectively. The optical gaps of $\mathrm{n}^{+}-, \mathrm{i}-$, and $\mathrm{p}^{+}$- layers, as determined by individual Tauc's plot, are 2.40 and 2.61 for Type I (or 2.65 for Type II), and $2.47 \mathrm{eV}$, respectively. The resistivities for the $\mathrm{n}^{+}$, Type-I $\mathrm{i}$, Type-II $\mathrm{i}$, and $\mathrm{p}^{+}$layers are $1.09 \times 10^{8}, 2.0 \times 10^{10}, 2.3 \times 10^{10}$, and $6.33 \times 10^{7} \Omega$. $\mathrm{cm}$, individually. The schematic optical-gap diagram under forward bias for the a-SiC:H DG TFLED is given in Fig. 1. The graded-gap $\mathrm{p}^{+}-\mathrm{i}$ and $\mathrm{i}-\mathrm{n}^{+}$junctions were achieved by terminating the $\mathrm{B}_{2} \mathrm{H}_{6}$ dopant gas and by adding the $\mathrm{PH}_{3}$ dopant gas during continuous depositions of a-SiC: $\mathbf{H}$ films, respectively. The thickness of these two graded regions were approximately equal to $30 \AA[10]$. Then, $5000-\AA \mathrm{Al}$ was thermally evaporated through a metal mask to define a circular device area of $1.13 \times 10^{-2} \mathrm{~cm}^{2}$. Finally, the device was annealed by using an ULVAC TA-7000 rapid thermal annealing (RTA) system at $300^{\circ} \mathrm{C}$ for $30 \mathrm{~s}$ initially and then at $150^{\circ} \mathrm{C}$ for $30 \mathrm{~min}$ sequentially in $\mathrm{H}_{2}$ ambient at a pressure of 110 torr, to improve the $\mathrm{Al}$ electrode $-\mathrm{n}^{+}$layer contact. The contact resistances between $\mathrm{n}^{+}$-layer and $\mathrm{Al}$ electrode before and after annealing are $6.66 \times 10^{5}$ and $1.38 \times 10^{5} \Omega \cdot \mathrm{cm}^{2}$, respectively.

\section{DeVICE OpERATION AND EXPERIMENTAL RESULTS}

The interface trapping states between $n^{+}-i$ and $p^{+}-i$ junctions could be greatly reduced due to the employed graded-gap regions in both junctions [10]. The injection currents for the carriers injected from the doped layers 


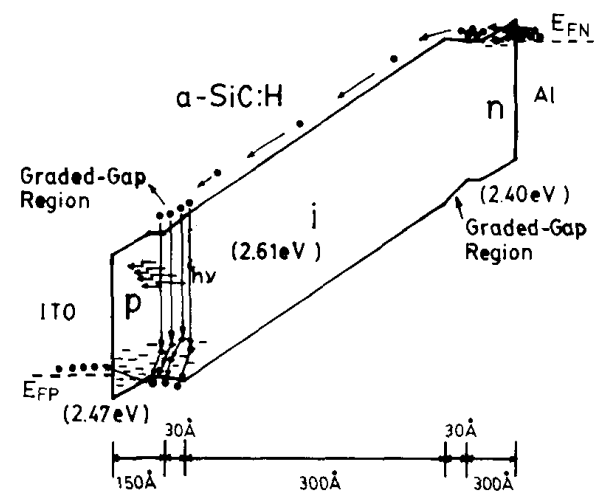

Fig. 1. The schematic optical-gap diagram of a-SiC:H Type I double graded-gap (DG) TFLED under forward bias.

into the i-layer could be improved due to the lowerings of effective energy barriers between the doped layers and the i-layer, since the doped layers have higher optical gaps than that in $[10](2.0 \mathrm{eV})$. The films deposited with a lower RF power density and the post-metallization annealing process intended to obtain a lower contact resistance also benefit to obtain a higher luminescence [11]. The utilization of wide optical-gap i-layers with more localized states increases the EL intensity and the emission energy [12]. These combined actions would improve the electron injection from the $\mathrm{n}^{+}$-layer into the conduction band of the i-layer and the hole traversing from the $\mathrm{p}^{+}$-layer into the localized states near the valence band of the i-layer. The electrons have a higher mobility than that of the holes. Therefore, the radiative recombination in the $\mathrm{p}^{+}$layer and the $\mathrm{i}$-layer near the $\mathrm{p}^{+}-\mathrm{i}$ interface, i.e., the EL intensity, can be improved [11].

Fig. 2 illustrates the brightness $B$ and injection current density $J$ versus applied forward bias $V$ for the Type I DG TFLED. A plausible explanation of the $J-V$ curve is as follows. Because there is a $1.6-\mathrm{eV}$ difference in electrochemical potential between the $\mathrm{n}^{+}$and $\mathrm{p}^{+}$regions, the injection current increases very rapidly with the forwardbias $V$ ranging from 0 to $1.6 \mathrm{~V}$. At $V=1.6 \mathrm{~V}$, a flat-band condition is achieved. For $1.6 \mathrm{~V}<V<8 \mathrm{~V}$, the injection current becomes a space-charge-limited current with trap [13], [14]. At $V=8 \mathrm{~V}$, the trap-filled limit is reached. As $V>8 \mathrm{~V}$ the current rises rapidly as a trap-free spacecharge-limited current [13], [14], and the brightness starts to increase.

The EL spectra of the TFLED's were measured by using a monochromater (ORIEL 77200), a photomultiplier (ORIEL 7070), and a PARC 5210 lock-in amplifier. The EL spectra for the devices with i-layer deposited by using three kinds of mole-fraction ratio, $x=$ $\left[\mathrm{C}_{2} \mathrm{H}_{2}\right] /\left(\left[\mathrm{SiH}_{4}\right]+\left[\mathrm{C}_{2} \mathrm{H}_{2}\right]\right)$, are given in Fig. 3. For the single graded $\mathrm{p}^{+}-\mathrm{i}$ junction (SG) TFLED [10], which emits red-orange light as detected by the naked eyes, the EL spectrum peaks at $710 \mathrm{~nm}$ with a full width at half maximum (FWHM) of $230 \mathrm{~nm}$. For the Type I DG TFLED, the EL spectrum peaks at $680 \mathrm{~nm}$ with a FWHM

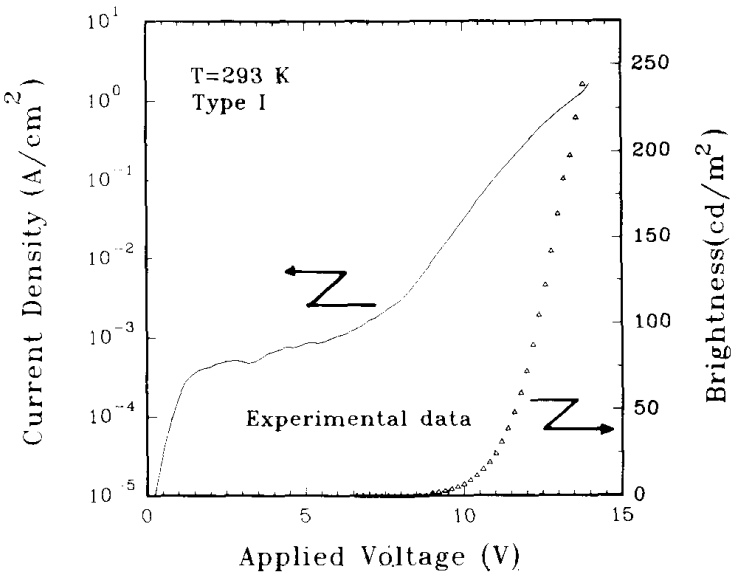

Fig. 2. The experimental brightness, and injection current versus applied forward biases for Type I DG TFLED.

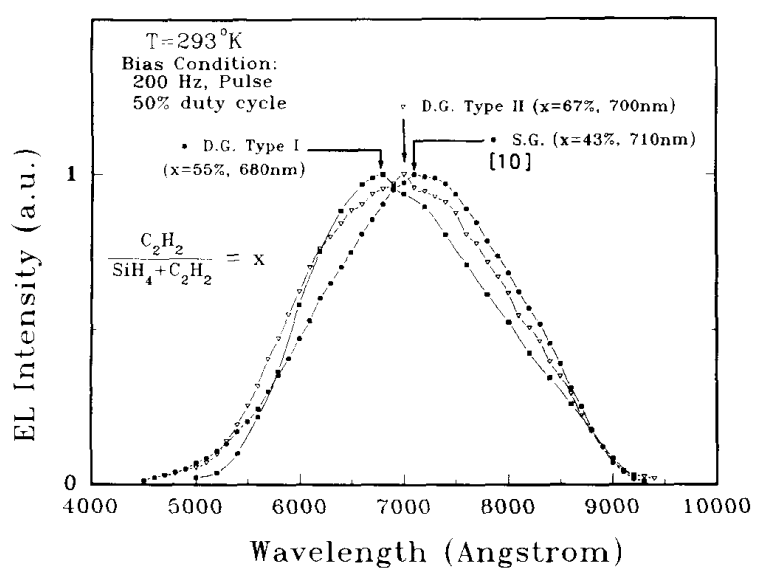

Fig. 3. The EL spectra of a-SiC:H double and single graded-gap TFLED's under $200-\mathrm{Hz}$ square-wave voltage with various peak-to-peak voltage $\mathrm{V}_{\mathrm{P}}=30 \mathrm{~V}$ for SG TFLED, $9 \mathrm{~V}$ for Type I DG TFLED, and $14 \mathrm{~V}$ for Type II DG TFLED.

of $215 \mathrm{~nm}$ and reveals an orange color. However, the EL spectrum of the orange Type II DG TFLED peaks at a longer wavelength $(700 \mathrm{~nm})$ and has a broader emission band $(F W H M=240 \mathrm{~nm}$ ) than that of the Type I DG TFLED. This could be primarily due to the wider energy range of the localized states in the a-SiC:H i-layer with a higher carbon content [15]-[17]. The EL spectra of these TFLED's peak at around $1.7 \mathrm{eV}$. This could be due to the radiative recombinations via the localized states with wide energy range in the amorphous $\mathrm{p}^{+}$- and i-layers.

Fig. 4 shows the brightness versus injection current density for the Type I and II DG TFLED's, HP (HewlettPackard) HLMP-8405 orange LED, and SG TFLED [10]. The DG TFLED's have much higher brightness than that of the SG TFLED, which is primarily due to the improvement of the injection currents, and hence, the increase of the radiative recombination probability. For the Type I DG TFLED, the brightness can be as high as $140 \mathrm{~cd} / \mathrm{m}^{2}$ 


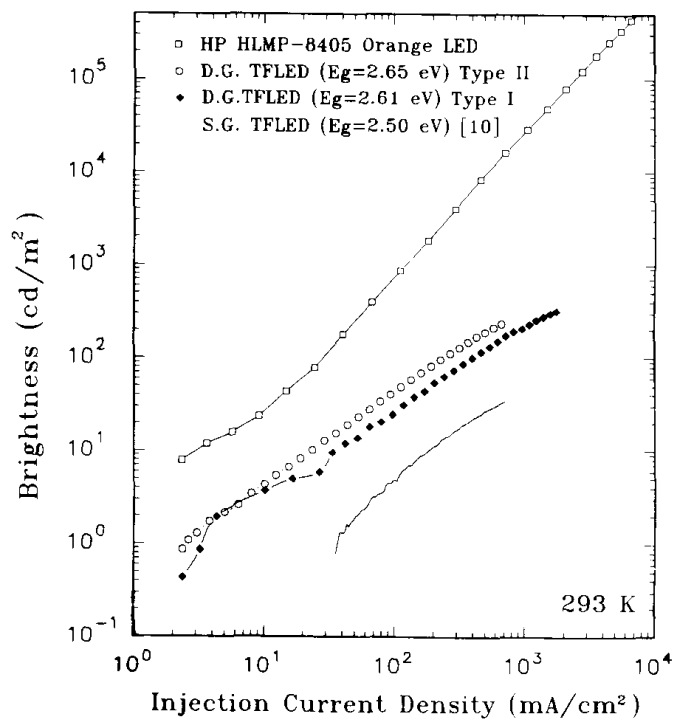

Fig. 4. Brightness versus injection current density for $\mathrm{DG}$ a-SiC:H TFLED's with i-layer optical gaps of 2.65 (Type II) and 2.61 (Type I) eV, single graded-gap (SG) a-SiC:H with an i-layer optical gap of $2.50 \mathrm{eV}$ [10], and an HP HLMP-8405 orange LED.

as calibrated by using an optometer (UDT S370) at a current density of $600 \mathrm{~mA} / \mathrm{cm}^{2}$. The Type II DG TFLED reveals a brightness of $207 \mathrm{~cd} / \mathrm{m}^{2}$ at the same injection current density. To our knowledge, this is the highest brightness ever reported for a-SiC:H TFLED's at the same injection current density [3]-[8], [10], [12]. The slight EL improvement of the Type II DG TFLED as compared to that of the Type I DG TFLED could be due to the wider energy range of the more localized states in the i-layer, which increases the recombination probability.

\section{CONCLUSION}

We have greatly improved the EL intensity of a-SiC:H $\mathrm{p}-\mathrm{i}-\mathrm{n}$ TFLED's by introducing the graded-gap $\mathrm{p}^{+}-\mathrm{i}$ and $\mathrm{i}-\mathrm{n}^{+}$junctions and have discussed its possible mechanism. At an injection current density of $600 \mathrm{~mA} / \mathrm{cm}^{2}$, the brightness of the Type II DG TFLED is as high as 207 $\mathrm{cd} / \mathrm{m}^{2}$. Its emitting light, as detected by the naked eyes, is orange.

\section{REFERENCES}

[1] J. I. Pankove and D. E. Carson, "Electroluminescence in amorphous silicon," Appl. Phys. Lett., vol. 29, pp. 620-622, 1976.

[2] A. J. Rhodes, P. K. Bhat, I. G. Austin, T. M. Searle, and R. A. Gibson, "Luminescence phenomena in a-Si:H p-i-n junction," $J$. Non-Cryst. Solid., vols. 59 and 60 , pp. 365-368, 1983.

[3] D. Kruangam, T. Endo, W. Guang-Pu, H. Okamoto, and Y. Hamakawa, "Visible-light injection-electroluminescent a-SiC $/ \mathrm{p}-\mathrm{i}-\mathrm{n}$ diode," Japan. J. Appl. Phys., vol. 24, pp. L806-L808, 1985.

[4] D. Kruangam et al., "Amorphous silicon-carbide thin film light emitting diode," Optoelectronics-Devices and Technologies, vol. 1, pp. 67-84, 1986.

[5] S. M. Paasche, T. Toyama, H. Okamoto, and Y. Hamakawa, "Amorphous-SiC thin film p-i-n light-emitting diode using amorphous-SiN hot-carrier tunneling injection layers," IEEE Trans. Electron Devices, vol. 36, pp. 2895-2902, 1989.

[6] Y. Hamakawa et al., "Toward a visible light display by amorphous SiC:H system," Optoelectronics-Devices and Technologies, vol. 4, pp. 281-294, 1989.

[7] D. Kruangam et al. "Visible light a-SiC multilayered thin film LED," in Extended Abstr. 18th Conf. Solid State Devices and Mater. (Tokyo), 1986, pp. 683-686.

[8] D. Kruangum, M. Deguchi, T. Toyama, H. Okamoto, and Y. Hamakawa, "Carrier injection mechanism in an a-SiC p-i-n junction thin-film LED," IEEE Trans. Electron Devices, vol. 35, pp. 957-965, 1988.

[9] H. Ihara and H. Nozaki, "Improvement of hydrogenated amorphous silicon n-i-p diode performance by $\mathrm{H}_{2}$ plasma treatment for i/p interface," Japan. J. Appl. Phys., vol. 29, pp. L2159-L2162, 1990 .

[10] J. W. Hong, N. F. Shin, T. S. Jen, S. L. Ning, and C. Y. Chang, "Graded-gap a-SiC:H p-i-n thin-film light-emitting diodes," IEEE Electron Device Lett., vol. 13, pp. 375-377, 1992.

[11] R. A. Street, J. C. Knights, and D. K. Biegelsen, "Luminescence studies of plasma-deposited hydrogenated silicon," Phys. Rev. B, vol. 18, pp. 1880-1891, 1978.

[12] J. Kanicki, Amorphous and Microcrvstalline Semiconductor Devices: Optoelectronic Devices. Boston: Artech House, 1991, ch. 6.

[13] J. Tauc, Amorphous and Liquid Semiconductors. New York: Plenum, 1974, ch. 5.

[14] H. P. Maruska, T. D. Moustakas, and M. C. Hicks, "Effects of optical stress on the properties of sputtered amorphous silicon solar cells and thin films," Solar Cells, vol. 9, pp. 37-51, 1983.

[15] P. Chaudhuri, S. Ray, A. K. Batabyal, and A. K. Barua "Properties of undoped and p-type hydrogenated amorphous silicon carbide films," Thin Solid Films, vol. 121, pp. 233-246, 1984.

[16] H. Kukimoto, Semiconductors and Semimetals, vol. 21D, J. I. Pankove, Ed. New York: Academic, 1984, ch. 12.

[17] S. R. Elliott, Physics of Amorphous Materials, 2nd ed. New York: Wiley, 1990, p. 399, ch. 6. 